\title{
Usage of frequency-controlled electric driver for hennery air ventilation systems
}

\author{
Mikhail Goryainov, ${ }^{1, *}$ \\ ${ }^{1}$ Technological service institute (affiliated branch) Don State Technical University, 41/1, ave. \\ Kulakova, 355035, Stavropol, Russia
}

\begin{abstract}
An uncontrolled electric driver is characterized with assonance with its features to technological process requirements. The most important characteristic of ventilation machines used in hennery air ventilation systems is the correlation between pressure and efficiency. To minimize the losses in running ventilators we have to control the ventilation process, thus to maintain the desired mode without departure from optimal value.
\end{abstract}

\section{Introduction}

First of all, electric drive efficiency depends on ventilator operating modes when sustain optimal conditions in the hennery. The most suitable firmware for this is a frequentlycontrolled driver.

Due to specific unit, henneries are expected to put large amount of poultry within the small place. That is why wrong operating of ventilation systems at the hennery leads to direct financial losses in case of diseases or even decrease of the whole livestock. Hennery air consists of carbon dioxide, excessive humidity and other suspended matters as skin, floss and feathers. Those must be constantly removed from the air, otherwise, the likelihood of inflectional diseases increases, productivity and weight gain decrease, whereas the rate of natural decrease excesses.

As for all living creatures, the circle of life of the birds includes outflux of carbon dioxide, warmth and moisture. Basing on that, we can conclude that main goal for hennery ventilation is evident: to provide required air purity, humidity level and temperature requirements.

On the other hand, there are some complicating factors, which makes construction of hennery air exchange system unique for each case:

- aggressive substances, outflowed from excrements (mainly ammonium compound, which accelerates corrosion of metal parts);

- significant volumes of moisture;

- high sensitivity of birds to temperature requirements and air leaks (speed range is from $0.2 \mathrm{~m} / \mathrm{s}$ to $0.4 \mathrm{~m} / \mathrm{s}$ );

- Significant dust outflows appeared when the forage is delivered;

- additional warmth generated by lamps when imitate daylight during winter;

- necessity to hold the excessive humidity level compared to the air outside (60-80\%).

\footnotetext{
*Corresponding author: mihailgoryainov@mail.ru
} 
Moreover, different breeds of birds need other animal welfare, which must be considered for hennery ventilation system:

1) $180 \mathrm{C}$ for hens

2) $160 \mathrm{C}$ for turkeys

3) 140C for waterfowl

Incubatory section also requires specific conditions, such as temperature range from 280-310C, with slight decrease as the poults grow.

Air ventilation system balances temperature and humidity, improves the air quality and removes exhaust air currents. Compared to ventilation systems at other animal husbandries, correct and stable work of the ventilation is more important as even little departure from optimal value causes sharp fall of manage productivity and outbreaks.

Designing ventilation systems is rather complicated. There are simplified options for designing on frequency (number of full air exchanges in the room within time unit) or on sanitary requirements for the unit (one bird or for 1 kilo of weight).

Ventilator productivity is the product of airspace and frequency number. In accordance with the other method, it is necessity of birds in certain airspace in the room.

Ventilator selection depends on design capacity rate. Two or more devices are installed in case one ventilator is not capable to provide the amount of air required.

As it was mentioned above, hennery ventilation systems are highly important as they provide safety, optimal volume, productivity and reproductive performance. Underestimating the problem means gross infringement of stockkeeping and culturing resulting in bird natural loss.

The original option for henneries is ventilation with drawn-in and vent lines connected to air heating system. Using rotary blowers allows to extract air with intaking nipples located in certain places in the room. Blower service at maximum is the reason for lowering endurance. Thus, motor capability falls and it breaks down as most of its parts are not suitable for such pressure. We have come to a conclusion that known ways of controlling blower operating modes are energy consuming, they also do not take into account link between air current and shaft torque. All mentioned above leads to uprating energy parameters of blower electric drive.

To improve the work of hennery ventilation system the electric drivers must be charged from frequency converters (FC) which also elongates driver endurance. FC using cuts costs on designing, installation work and simplifies maintenance of ventilation systems. All of this is accessible due to easing the control scheme, thereby power consuming is lower. Using frequency-controlled driver (FCD) provides driver protection from overcharging as well as asymmetrical load. FCD allows to avoid additional safety system in case of voltage variations as well abnormal and crash conditions. Rotation frequency control also allows to start the driver softly limiting the motor starting currents. This elongates the repair interval of firmware. As reaching required parameters and optimal energy mode for controlled drivers is possible with converting electrical parameters (voltage and frequency), efficiency coefficient and power loss depend on the way of controlling and pressure rate. Foremost, electric driver efficiency depends on blower operating modes when maintain optimal conditions in henneries. Non-FCD systems control air induction with slide gates or shifting angle of fan blades. This way causes power overuse as, shifting air induction, the system consumes literally the same amount of energy. As a result, underloaded system takes the same amount of energy.

So, the issues, which must be considered in first line in order to improve operating characteristics and decreasing energy demands, are closely connected with development of the following methods:

Applicability of power saving and enhancing of power efficiency at henneries is determined by high prices for power resources. 
It is clear that aggregate consume costs is a sum of semi-fixed and semi-variable costs.

Notations introduced:

$\mathrm{I}_{\text {consto }}, \mathrm{I}_{\text {const } 1}$ - semi-fixed costs independent from amount of energy and consuming time in initial and compared periods.

$I_{\text {var } 0}, I_{\text {var } 1}-$ semi-variable costs dependent on amount of energy and consuming time in initial and compared periods.

$I_{\text {about } 0}, I_{\text {about } 1}$ - aggregate costs in initial and compared periods.

$\mathrm{t}_{0}, \mathrm{t}_{1}$ - operating time in initial and compared periods.

When talking about innovative, modernization, repairing, preventive activities, the two processes co-exist. The first is connected to producing costs within "pre-existing" process (initial period), which mathematically can be described as a sum of semi-fixed and semivariable costs. This gives a result of aggregate operating and distribution costs $\left(\mathrm{I}_{\text {about } 0}\right)$ connected with maintaining of replaced firmware in time $\mathrm{t}$ :

$$
I_{\text {about } 0}=I_{\text {const } 0}+I_{\text {var } 0} \cdot t
$$

The second is connected to producing costs within the "new" process (compared period), which mathematically can be described as a sum of semi-fixed and semi-variable costs. This gives a result of aggregate operating and distribution costs $\left(I_{\text {about } 0}\right)$ connected with maintaining of replaced firmware in time $t$ :

$$
I_{\text {about } 1}=I_{\text {const } 1}+I_{\text {var1 }} \cdot t
$$

When talking about innovative, modernization and so on activities aimed to power saving and enhancing energy efficiency, the two processes cooperate.

Figure 1 shows how this cooperation works. Process, shown in the graph $\mathrm{OAA}_{1} \mathrm{~A}_{2} \mathrm{~A}_{3}$, expresses movement of aggregate costs in terms of the "old" quality, basic system, basic process within the initial period. Aggregate sum of semi-fixed costs is defined on y-axis by OC number and consists of costs for buying "old", basic equipment OA and costs for replacing broken parts of equipment as well as costs for maintenance inspection $\mathrm{AB}$ and BC. As the number of this activities might be more than one within the certain time period $\mathrm{t}$, each stage is shown as a step, which number is practically unlimited.

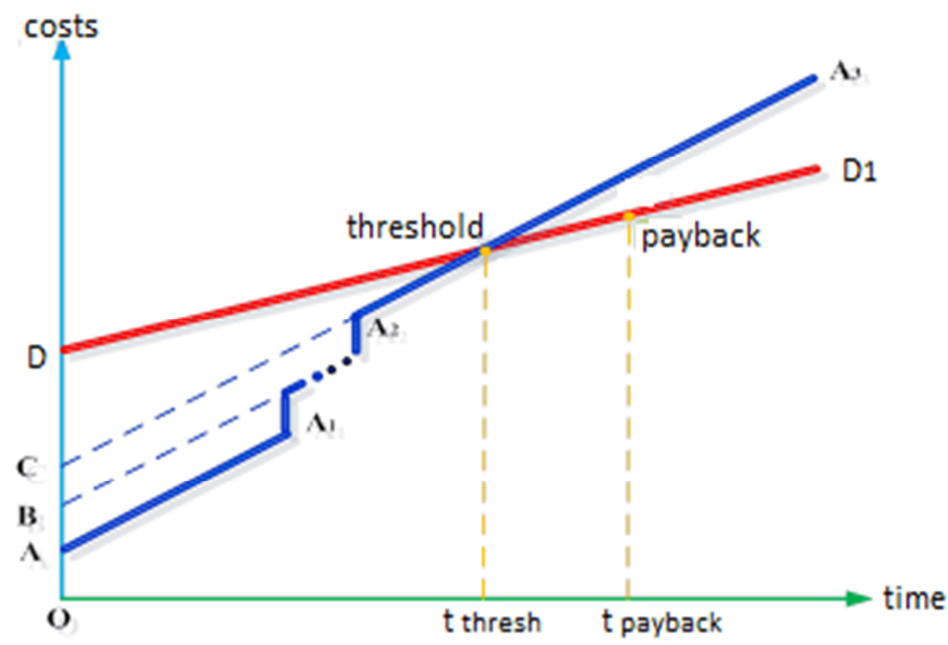

Fig. 1. Defining power saving "turn-out" and pay-off period for power saving costs.

Angled line $\mathrm{AA}_{1} \mathrm{~A}_{2} \mathrm{~A}_{3}$ is the graph of semi-variable costs, which describes their shift, sum and dependence on the amount of consumed energy when maintaining the "old" 
equipment in the initial period.

So, according to the formula (1) the graph $\mathrm{AA}_{1} \mathrm{~A}_{2} \mathrm{~A}_{3}$ is a shift of aggregate costs within the initial period $t$.

The process, shown in the graph ОДД 1 is a shift of aggregate costs in terms of "new" quality, modernized system and new process within the compared period.

Sum total of semi-fixed costs is defined on y-axis by OD number and consists of the costs for buying new equipment in compared period. We suggested the costs for replacing broken parts of equipment, preventive inspection, control of equipment state are zero as the firmware is "new", more modern and of high quality, so it works without replacement the whole maintenance period within compared period. But if such costs appear, the same "ladder" as for line $\mathrm{AA}_{1} \mathrm{~A}_{2} \mathrm{~A}_{3}$ appears. Anyway, for the main issue this one has no importance.

The straight line $\mathrm{DD}_{1}$ is the graph of semi-variable costs, which describes their shift, sum and dependence on the amount of consumed energy when maintaining the "old" equipment in the compared period.

The movement of "old" and "new" aggregate costs for initial and compared periods comes to balance in E point. Mathematically, this state means equality for aggregate costs $\mathrm{I}_{\text {about } 0}$ (formula (1)) and $\mathrm{I}_{\text {about } 1}$ (formula (2)). $\mathrm{E}$ is the starting point of power saving, the "new" process, formation of a "new" modernized system, so called "edge" of power saving not only the separate components, but the system in general. Obviously, power saving of the "new" system starts when the new equipment starts to consume power. However, that is only part of how the system orks because the costs for buying new firmware are still uncovered.

Mathematically it is implied as "edge" time, where power saving starts.

Calculations for power saving "edge" time:

$$
\begin{gathered}
I_{\text {about0 }}=I_{\text {const0 }}+I_{\text {var0 } 0} \bullet t_{\text {thresh }}= \\
=I_{\text {about } 1}=I_{\text {const1 }}+I_{\text {var } 1} \cdot t_{\text {thresh }}
\end{gathered}
$$

That is why

$$
t_{\text {thresh }}=\frac{I_{\text {const } 1}-I_{\text {const } 0}}{I_{\text {var } 0}-I_{\text {var } 1}}
$$

The resulted formula for power saving "edge" time is key, fundamental and, moreover, new feature in theory of power saving.

The graph on Fig. 2 also has point $\mathrm{O}_{\kappa}$ - pay-off period for new equipment ( $\mathrm{t}_{\text {payback }}$ ). According to the graph, pay-off period for new or modernized equipment is always longer than "edge" time $\left(\mathrm{t}_{\text {thresh }}\right)$. As it is known, pay-off period for innovations, modernization, replacement and so on is calculated the following way:

$$
t_{\text {payback }}=\frac{I_{\text {const1 }}}{I_{\text {about } 0} I_{\text {about } 1}}
$$

where $I_{\text {about } 0}, I_{\text {about } 1}^{\prime}$ - aggregate costs per year for "old" existing and "new" modernized variants (the average number of workdays is 247).

Traditional formula (5) for pay-off period is not derived from disadvantages. For instance, aggregate costs which describe costs for buying firmware presented both in numerator and denominator. This fact falsifies length of pay-off period because pay-off for firmware depends only on saving consumed energy.

To our mind, pay-off period for modernized equipment can be calculated from correlation of equipment price (aggregate costs $I_{\text {const } 1}$ ) and saving rate within a time unit (remainder between variable costs $\mathrm{I}_{\mathrm{var} 0}$ end $\mathrm{I}_{\mathrm{var} 1}$ ): 


$$
t_{\text {payback }}=\frac{I_{\text {const1 }}}{I_{\text {var } 0}-I_{\text {var } 1}}
$$

Having compared power saving "edge" time (formula 4) and pay-off period for modernized equipment (formula 6), we have come to a conclusion that pay-off period for modernized equipment is longer than power saving "edge" time.

Practically, aggregate semi-fixed expenses and aggregate semi-variable costs can be presented as separate spending patterns:

- aggregate semi-fixed expenses of initial period are sum of itemized semi-fixed costs ( ( $_{\text {const i } 0)}$ )

$$
I_{\text {const0 }}=\sum_{i}^{a} I_{(\text {consti }) 0}
$$

- aggregate semi-fixed expenses of compared period are sum of itemized semi-fixed costs ( (const i) 1$)$

$$
I_{\text {const1 }}=\sum_{i}^{b} I_{(\text {const } i) 1}
$$

- aggregate semi-variable expenses of initial period are sum of itemized semi-variable costs (I $\left.\mathrm{I}_{(\mathrm{var} \text { i) } 0}\right)$

$$
I_{\mathrm{var} 0}=\sum_{i}^{c} I_{(\mathrm{var} i) 0}
$$

- aggregate semi-variable expenses of compared period are sum of itemized semi-variable

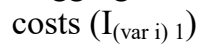

$$
I_{\mathrm{var} 1}=\sum_{i}^{d} I_{(\operatorname{var} i) 1}
$$

Taking account for the formulae (7-10) power saving "edge" time is:

$$
t_{\text {thresh }}=\frac{\sum_{i}^{b} I_{(\mathrm{const} i) 1}-\sum_{i}^{a} I_{(\mathrm{const} i) 0}}{\sum_{i}^{c} I_{(\mathrm{var} i) 0}-\sum_{i}^{d} I_{(\mathrm{var} i) 1}}
$$

So, resulting formulae ((4), (6), (11)) help solve other problems such as:

- knowing power saving "edge" time, we can calculate required amount of aggregate expenses for innovative and modernization activities;

- knowing the amount of invest money for innovative and modernized firmware, we can calculate amount of semi-variable expenses in compared period dealing with different numbers of power saving "edge" time and so on.

Foremost, electric driver efficiency depends on blower operating modes when maintain optimal conditions at the hennery.

Figures $2 \mathrm{a}$ and $\mathrm{b}$ show graphic charts $\eta$ and $\cos \varphi_{1}$ in dependence on voltage with two numbers of driver shaft power $P_{\text {nominal }}$ and $0,5 P_{\text {nominal }}$. Thus, when the shaft power decreases to $0,5 P_{\text {nominal }}$, efficiency coefficient also decreases to $8 \%$, and $\cos \varphi_{1} 24 \%$. If the pressure is lower, then due to voltage decrease efficiency coefficient and $\cos \varphi_{1}$ can be increased. This is because current $\Phi \mathrm{m}$, no-load current and magnetic losses are also decreased. Figure 2 shows that each pressure matches its own voltage rate when the constant losses are equal to variable ones. Lowering pressure must be accompanied by lowering voltage. In that way certain pressure matches the voltage when $\cos \varphi_{1}$ is at its maximum. 

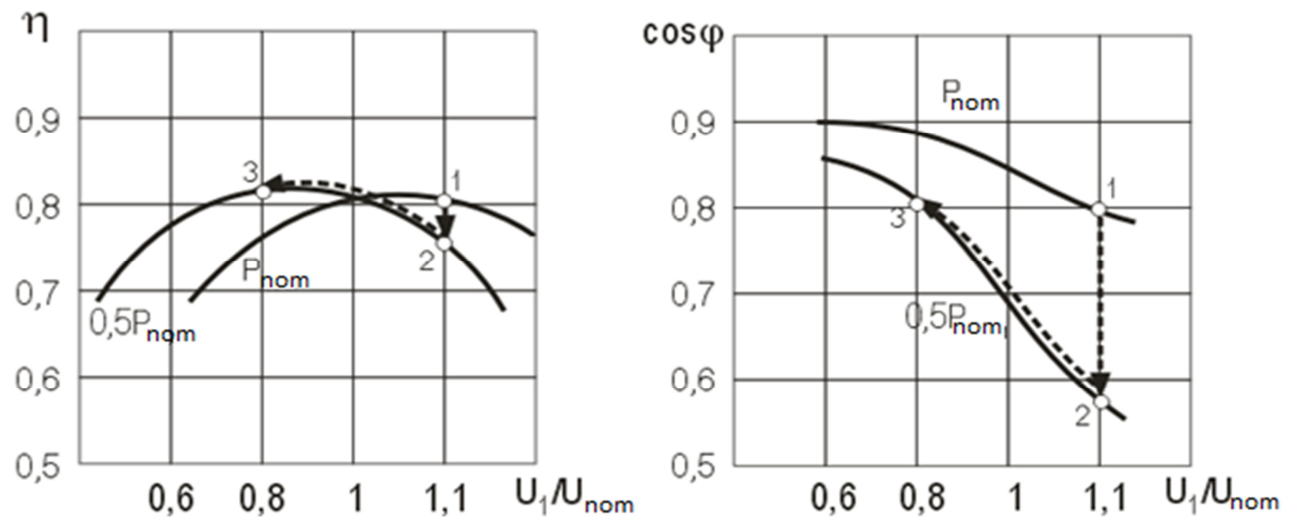

Fig. 2. Dependence $\eta(a)$ and $\cos \varphi_{1}(b)$ on charging voltage with nominal and decreased pressure.

During the experiment the following conclusion has been made: when the pressure is decreased to $0,5 P_{\text {nominal }}$ (Fig. 2, $a$ ), efficiency coefficient decreases to the number in point 2 . Then, when the voltage is decreased to $0,8 U_{\text {nominal }}$ efficiency coefficient increases to the number in point 3 . The same changes happen with $\cos \varphi$ when the pressure is lowered. Fig. 3 presents diagrams of consuming energy when air current is regulated with sliders (diagram 1) and with FCD (diagram 2).

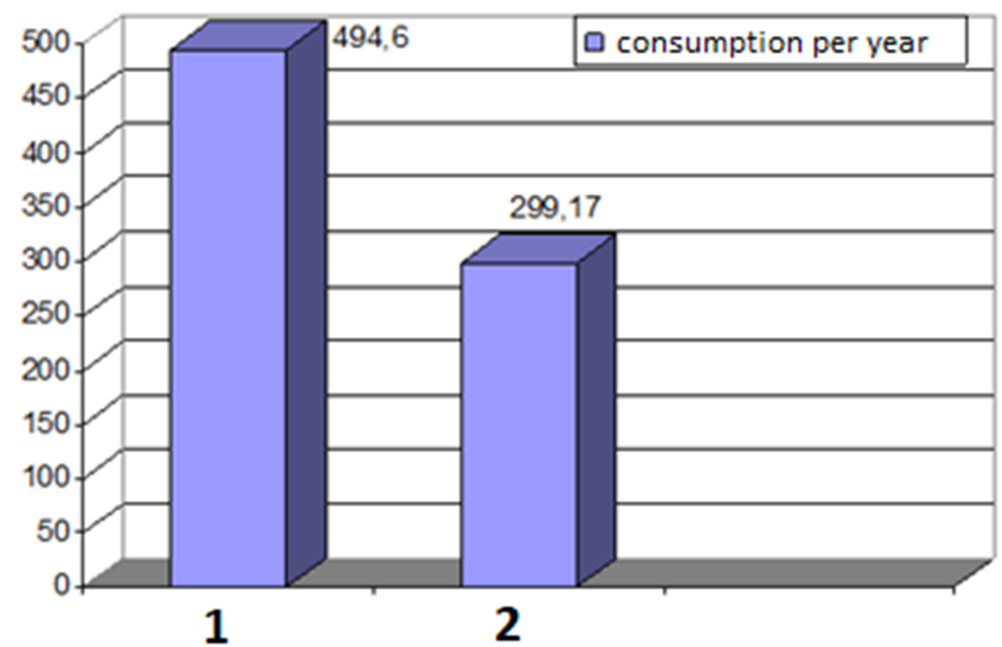

Fig. 3. Diagrams of consuming energy by blowers within a year with sliders (1) and FCD (2).

To conclude, we would like to notice that amount of consumed energy, when air current is regulated by sliders, is on average $24-28 \%$ larger than for FCD regulated air current.

\section{References}

1. I. Yun, Printed Electronics, Current Trends and Applications (2016) DOI: 10.5772/61800, ISBN: 978-953-51-2527-3, ISBN: 978-953-51-2301-9, ISBN: 978953-51-6679-5

2. P. Breeze, Power Generation Technologies (Newnes, 2019) ISBN: 9780128182550 , 9780081026311

3. I. Yahyaoui, Advances in Renewable Energies and Power Technologies (Elsevier 
Science, 2018) ISBN: 9780128129593 , ISBN: 9780128132173

4. Energy Efficiency and Renewable Energy Handbook (CRC Press, 2018) ISBN 9781138749115 - CAT\# K32717

5. Distributed Generation: The Power Paradigm for the New Millennium (CRC Press 2019) ISBN 9780367397197 - CAT\# K449211

6. Yixiang Zhang, Congcong Xiao, Guanghui Zhou, Willingness to pay a price premium for energy-saving appliances: Role of perceived value and energy efficiency labeling (Elsevier BV, 2019) DOI: 10.1016/j.jclepro.2019.118555

7. V. Máša, P. Stehlík, M. Touš, M. Vondra, Key pillars of successful energy saving projects in small and medium industrial enterprises (Elsevier BV, 2018) DOI:10.1016/j.energy.2018.06.018

8. A. Prashar, Towards sustainable development in industrial small and Medium-sized Enterprises: An energy sustainability approach (Elsevier BV,2019) DOI:10.1016/j.jclepro.2019.07.045

9. Xiaoyan Qi, Pibin Guo, Yanshan Guo, Xiuli Liu, Xijun Zhou, Understanding energy efficiency and its drivers: An empirical analysis of China's 14 coal intensive industries (Elsevier BV, 2019) DOI:10.1016/j.energy.2019.116354

10. Xiaofan Zhao, Huimin Li, Liang Wu, Ye Qi, Enterprise-level amount of energy saved targets in China: weaknesses and a way forward (Elsevier BV, 2016) DOI:10.1016/j.jclepro.2016.04.116

11. J. Henriques, J. Catarino, Motivating towards energy efficiency in small and medium enterprises (Elsevier BV, 2016) DOI: 10.1016/j.jclepro.2016.08.026

12. L. Zhang, R. Long, H. Chen, X. Huang, Performance changes analysis of industrial enterprises under energy constraints (Elsevier BV, 2018) DOI:10.1016/j.resconrec.2018.04.032

13. J. Fresner, F. Morea, C. Krenn, J. Aranda Uson, F. Tomasi, Energy efficiency in small and medium enterprises: Lessons learned from 280 energy audits across Europe (Elsevier BV, 2016) DOI:10.1016/j.jclepro.2016.11.126

14. A. Tallini, L. Cedola, Evaluation Methodology for Energy Efficiency Measures in Industry and Service Sector (Elsevier BV, 2016) DOI:10.1016/j.egypro.2016.11.069

15. M. Song, S. Wang, Measuring environment-biased technological progress considering energy saving and emission reduction (Elsevier BV, 2017) DOI:10.1016/j.psep.2017.08.042 